\title{
CYCLICITY OF ELEMENTARY POLYCYCLES WITH FIXED NUMBER OF SINGULAR POINTS IN GENERIC $k$-PARAMETER FAMILIES
}

\author{
P. I. KALEDA AND I. V. SHCHUROV
}

\begin{abstract}
An estimate is found for the number of limit cycles arising from polycycles in generic finite-parameter families of differential equations on the two-sphere. It is proved that if the polycycles have a fixed number of singular points and all the singular points are elementary, then an estimate of cyclicity holds true, which is polynomial in the number of parameters of the family.
\end{abstract}

\section{$\S 1$. INTRODUCTION AND STATEMENT OF THE MAIN RESULT}

1.1. Motivation and the preceding results. In the present paper, we discuss the Hilbert-Arnol'd problem, which is closely related to Hilbert's 16th problem. In the latter problem, it is required to estimate the number of limit cycles of a polynomial vector field on the plane. Arnol'd [1] proposed to consider not polynomial families, but arbitrary generi 1 finite-parameter families of smooth differential equations on the two-sphere with compact base of parameters, and he formulated a number of conjectures.

One of them turned out to be incorrect, but nevertheless it led Il'yashenko [2] to the statement of the following problem: Show that for every such family, the number of limit cycles admits a uniform estimate for all values of the parameter.

Using compactness arguments going back to Roussarie 7, one can reduce the above problem to estimating the number of cycles arising from bifurcations of the so-called polycycles, i.e., separatrix polygons. The latter problem is called the (local) HilbertArnol'd problem. (See Problem 1 below.)

Rigorous definitions and the statement of the problem are presented below.

Definition 1.1. A polycycle $\gamma$ of a vector field on the sphere $\mathbb{S}^{2}$ is a cyclically ordered collection of vertices, i.e., singular points $p_{1}, \ldots, p_{n}$ (possibly, with repetition), and arcs of trajectories $\gamma_{1}, \ldots, \gamma_{n}$ (without repetition) connecting the vertices in the following order: the $j$ th arc connects the vertices $p_{j}$ and $p_{j+1}$, where $j=1, \ldots, n$ and $p_{n+1}:=p_{1}$.

A polycycle is said to be nontrivial if it contains at least one singular point. The maximum number of cycles that can arise from a perturbation of a polycycle is called the cyclicity of the polycycle. The rigorous definition is as follows.

Definition 1.2. Consider a $k$-parameter family of vector fields on the sphere:

$$
\{\dot{x}=v(x, \varepsilon)\}_{\varepsilon \in B^{k}}, \quad x \in \mathbb{S}^{2},
$$

2010 Mathematics Subject Classification. Primary 34C07.

Key words and phrases. Number of limit cycles, polycycle, Hilbert's sixteenth problem, HilbertArnol'd problem.

Partially supported by RFBR (grant no. 7-01-00017-a), and RFBR/CNRS (grant no. 05-01-02801CNRSa).

${ }^{1}$ Genericity is understood here in the topological sense. 


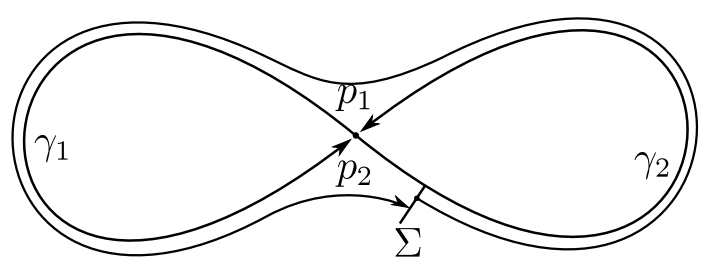

Figure 1. A polycycle and the Poincaré map.

where $B^{k}$ is the unit ball in $\mathbb{R}^{k}$. Assume that the system has a polycycle $\gamma$ for $\varepsilon=\varepsilon_{*} \in B^{k}$. Let $\mu$ be the minimum integer with the following property: there exist neighborhoods $U$ and $V$ such that

$$
\mathbb{S}^{2} \supseteq U \supset \gamma, \quad B^{k} \supseteq V \ni \varepsilon_{*},
$$

and for each $\varepsilon \in V$ the neighborhood $U$ contains at most $\mu$ limit cycles of the field $v(\cdot, \varepsilon)$. Then $\mu$ is called the cyclicity of the polycycle $\gamma$ in the family $\{v(x, \varepsilon)\}_{\varepsilon \in B^{k}}$.

Definition 1.3. The bifurcation number $B(k)$ is the maximum cyclicity of a nontrivial polycycle in a generic $k$-parameter family of $C^{\infty}$-smooth vector fields.

We observe that the number $B(k)$ depends only on the number of parameters in the family.

Problem 1 (Hilbert-Arnol'd problem). Prove that the bifurcation number $B(k)$ is finite for each finite $k$, and find an upper bound for $B(k)$.

This problem remains open.

Definition 1.4. A singular point of a vector field $v$ on the two-sphere $\mathbb{S}^{2}$ is said to be elementary if the linearization of $v$ at that point has at least one nonzero eigenvalue. A polycycle is elementary if all vertices of the polycycle are isolated elementary singular points.

The Hilbert-Arnol'd problem was solved for the case of an elementary polycycle. Yu. Il'yashenko and S. Yakovenko made the first step, and the next step was made by V. Kaloshin.

Definition 1.5. The maximum cyclicity of a nontrivial elementary polycycle in a generic $k$-parameter family of $C^{\infty}$-smooth vector fields is called the elementary bifurcation number and is denoted by $E(k)$.

Theorem 1.6 (Il'yashenko and Yakovenko [4]). For each positive integer $k$, the number $E(k)$ is finite.

Theorem 1.7 (V. Kaloshin [5]). For each positive integer $k$, we have

$$
E(k) \leq 2^{25 k^{2}} .
$$

Theorem 1.7 yields the first explicit estimate for the cyclicity of a polycycle in a family with an arbitrary number of parameters. However, estimate (1.1) appears to be greatly overstated.

1.2. Statement of the main result of the paper. It turns out that if the number of singular points of the system is fixed, then the cyclicity grows not faster than a certain polynomial in the number of parameters of the family. Our main result is Theorem 1.8, which suggests an explicit estimate of such a form. 
Theorem 1.8. The maximum cyclicity of a nontrivial elementary polycycle with $n$ vertices in a generic $k$-parameter family is bounded by the number

$$
E(n, k) \leq C(n) k^{3 n},
$$

where $C(n)=2^{5 n^{2}+20 n}$.

In the present paper, we use techniques developed by Il'yashenko jointly with Yakovenko [4] and substantially elaborated by Kaloshin [5].

1.3. Structure of the paper. In $\oint 2$ we describe a strategy for solving problems of this type and give necessary formulations. In Subsection 2.7, we clarify the difference between the techniques developed by Kaloshin and those developed by Il'yashenko and Yakovenko. There, we also describe the main idea that allowed us to obtain sharper estimates and to prove Theorem 1.8. Finally, the necessary computations are presented in $₫ 3$.

The authors are grateful to Yu. Il'yashenko for the statement of the problem and help in their work, to V. Kaloshin for consultations, and to D. Ryzhov who has read the manuscript and made valuable remarks on the text.

\section{§2. Existence of the NUmber $E(n, k)$ And its estimate}

A complete description of the techniques for solving similar type problems is beyond the scope of the present paper; it can be found in [5] and [4. In this section, we describe the strategy of the proof; largely we follow [5] and [4].

2.1. Plan of the proof. We consider a generic $k$-parameter family of vector fields on the plane depending on the parameter $\varepsilon$ and assume that for $\varepsilon=\varepsilon_{*}$ the corresponding system possesses a nontrivial polycycle. Here, we use a standard trick and consider the Poincaré map. After that, the problem of estimating the number of limit cycles of a differential equation reduces to estimating the number of isolated fixed points of the Poincaré map (see Subsection 2.2).

We look for the fixed points as for solutions of a special system of equations, which is called the basic system. (The system also depends on the parameter $\varepsilon$.) The further plan is to use sequentially a number of transformations and bring the basic system to a form admitting an effective estimate for the number of solutions. When doing so, we control the estimate for the number of solutions during each transformation (see Figure 2).

Using finitely smooth changes of coordinates, we bring the initial family of vector fields to a special simple form in neighborhoods of singular points. This allows us to write the equations of the basic system in the normalized form (see Subsection 2.3).

The equations involve singular functions, which are difficult for study. However, solutions of such equations, in turn, satisfy the so-called Pfaffian equations, i.e., differential equations with polynomial coefficients (see Subsection 2.4). Therefore, the number of solutions of the basic system is estimated by the number of solutions of a new functionalPfaffian system, in which equations with singular functions are replaced by Pfaffian differential equations. This allows us to eliminate the singular functions.

Below, we use a trick known as Khovanskiı's reduction. As a result, the functionalPfaffian system is replaced by a (finite) collection of purely functional systems having a special form and containing no singular equations. Furthermore, the number of solutions of the initial system does not exceed the sum of the numbers of solutions of the systems in the collection (see Subsection 2.5).

An analog of Bezout's theorem is applicable to such systems: this is the BezoutKaloshin theorem. The latter allows us to give an explicit estimate for the number of 


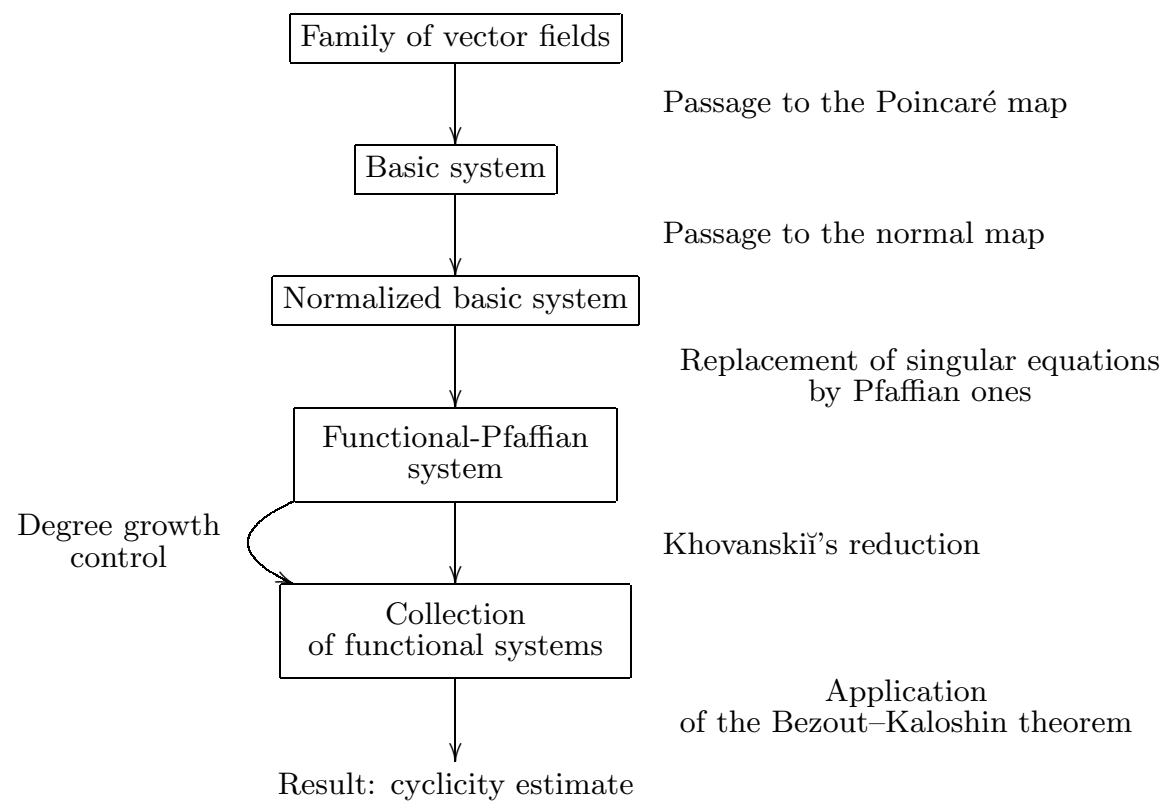

FiguRe 2. Strategy of the proof.

solutions and, simultaneously, for the number of limit cycles in the initial family (see Subsection 2.6).

In order to complete the proof, it only remains to calculate the degrees of the equations in the systems to which the Bezout-Kaloshin theorem is applied. This is done in 93 ,

2.2. The basic system. We denote by $n$ the number of vertices of a nontrivial elementary polycycle, and we denote by $k$ the number of parameters of the family. Our problem is to estimate the number of limit cycles arising from a given polycycle in terms of $n$ and $k$.

A polycycle $\gamma$ is said to be monodromic if the phase space contains a half-interval $\Sigma$ such that an end of $\Sigma$ lies on $\gamma, \Sigma$ is transversal to the vector field, and the Poincaré map (the first return map; see Figure 3) is defined on $\Sigma$. Such a half-interval $\Sigma$ is briefly called a transversal to the polycycle $\gamma$.

We assume that the polycycle is monodromid 2 and consider the corresponding Poincaré map $\Pi$.

Let $\Sigma_{j}^{\text {in }}$ and $\Sigma_{j}^{\text {out }}$ be transversals to the polycycle in the vicinity of the $j$ th vertex $(j=1, \ldots, n)$ such that the correspondence mappings

$$
\Delta_{j}: \Sigma_{j}^{\text {in }} \rightarrow \Sigma_{j}^{\text {out }} \quad \text { and } \quad f_{j}: \Sigma_{j}^{\text {out }} \rightarrow \Sigma_{j+1}^{\text {in }}
$$

are defined (where we put $\Sigma_{n+1}^{\text {in }}:=\Sigma_{1}^{\text {in }}$ ) and the mapping $\Pi$ is the composition

$$
\Pi=f_{n} \circ \Delta_{n} \circ \cdots \circ f_{1} \circ \Delta_{1}
$$

(see Figure 3).

\footnotetext{
${ }^{2}$ Remark 2.9 translates the construction to the case of nonmonodromic polycycles.
} 


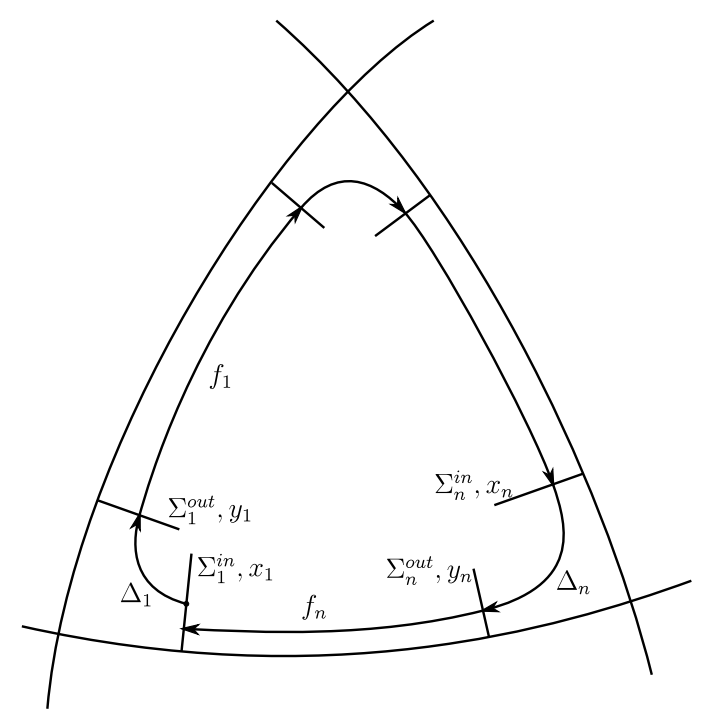

Figure 3. The Poincaré map of the polycycle.

If closed trajectories arise from a perturbation of the polycycle, then they correspond to fixed points of the mapping $\Pi$. They can be found as solutions of the basic system:

$$
\left\{\begin{array}{l}
y_{j}=\Delta_{j}\left(x_{j}, \varepsilon\right), \\
x_{j+1}=f_{j}\left(y_{j}, \varepsilon\right), \quad j=1, \ldots, n, \\
x_{n+1}=x_{1},
\end{array}\right.
$$

where $x_{j}$ is the coordinate on the "input" transversal $\Sigma_{j}^{\text {in }}$ of the $j$ th vertex, $y_{j}$ is the coordinate on the "output" transversal $\Sigma_{j}^{\text {out }}$ of the $j$ th vertex, and

$$
\varepsilon=\left(\varepsilon_{1}, \ldots, \varepsilon_{k}\right)
$$

is the vector of parameters.

2.3. Normal forms of local families. If an elementary polycycle can generate limit cycles, then topological arguments imply that the only singular points on the polycycle are saddles and saddle-nodes. Using finitely smooth changes of variables, we bring local families presenting perturbations of saddles and saddle-nodes to normal forms described in 3 . In this section, we formulate the necessary facts.

The saddles split into two classes having distinct normal forms: resonant and nonresonant saddles; the first case corresponds to the rational quotient $\frac{\mathrm{n}}{\mathrm{m}}$ of eigenvalues (in this case, we speak about a resonance of type $\mathrm{n}: \mathrm{m}$ ). The second case corresponds to an irrational quotient of eigenvalues.

Let $\mu$ be a positive integer, and let $\lambda=\left(\lambda_{0}, \ldots, \lambda_{\mu}\right)$ be a multidimensional parameter. We introduce the following notation:

$$
\begin{aligned}
u & =x^{m} y^{n}, \\
P_{\mu}(u, \lambda) & = \pm u^{\mu}\left(1+\lambda_{\mu} u^{\mu}\right)+W_{\mu-1}(u, \lambda), \\
Q_{\mu}(x, \lambda) & =x^{\mu+1}+\lambda_{\mu} x^{2 \mu+1}+W_{\mu-1}(x, \lambda),
\end{aligned}
$$

where

$$
W_{\mu-1}(x, \lambda)=\lambda_{0}+\cdots+\lambda_{\mu-1} z^{\mu-1}
$$


The normal form of a resonant saddle in a finite-parameter family of vector fields looks like this:

$$
\left\{\begin{array}{l}
\dot{x}=x\left(\frac{\mathrm{n}}{\mathrm{m}}+P_{\mu}(u, \lambda)\right), \\
\dot{y}=-y
\end{array}\right.
$$

(see [4, Table 1]). The number $\mu$ depends on the initial saddle and is called the multiplicity of the saddle; we denote by $S_{\mu}$ saddles of multiplicity $\mu$.

Remark 2.1. Actually, the normal form (2.3) occurs when the germ of the vector field for $\varepsilon=\varepsilon_{*}$ has no nontrivial formal first integral (see [3]). This condition determines a set of infinite codimension in the space of smooth hyperbolic resonant germs of vector fields.

For a singular point of saddle-node type, we denote by $\mu+1$ the usual multiplicity of the singular point (i.e., the maximum number of singular points into which the singular point "scatters" under a small perturbation).

In a finite-parameter family of vector fields, the normal form of a saddle-node of multiplicity $\mu+1$ looks like this:

$$
\left\{\begin{array}{l}
\dot{x}=Q_{\mu}(x, \lambda) \\
\dot{y}=-y
\end{array}\right.
$$

(see [4, Table 1]).

Remark 2.2. Actually, the normal form (2.4) occurs when the Lojasiewicz condition is fulfilled (when approaching the singular point, the length of the vector field decreases not faster than a certain power of the distance to the point); see [4. This condition determines a set of infinite codimension in the space of smooth saddle-node germs of vector fields.

Using the normal forms indicated above, we may assume that the basic system (2.1) is normalized, i.e., each function $\Delta_{j}$ corresponds to a singular point written in the normal form.

With the help of the normalized basic system, a $k$-parameter family of vector fields determines a $k$-parameter family of collections of finitely smooth functions $f_{j}$. As a result, we obtain the following proposition.

Proposition 2.3. Suppose that the functions $f_{j}$ in the basic system (2.1) are generic. Then the maximum cyclicity in Theorem 1.8 does not exceed the number of solutions of system (2.1) that are close to zero.

Remark 2.4. A precise description of the set of generic collections of finitely smooth functions $f_{j}$ will be given below; see Definition 2.17 .

2.4. Pfaffian equations and the functional-Pfaffian system. The normal forms (2.3) and (2.4) can be integrated in quadratures, and the correspondence mappings for them can be computed explicitly. These mappings are determined by transcendental functions and in general are not everywhere defined (see, e.g., [4, §1.1]). However, the mappings satisfy Pfaffian equations, i.e., differential equations with polynomial coefficients (loc. cit.). We can replace the singular equations

$$
y_{j}-\Delta_{j}\left(x_{j}, \varepsilon\right)=0
$$

by the Pfaffian equations satisfied by their left-hand sides. We observe that the number of solutions of the resulting system (the latter is called the functional-Pfaffian system) does not exceed the number of solutions of the corresponding basic system (2.1).

We now present the necessary formulations. 
Lemma 2.5 (Il'yashenko and Yakovenko [4]). The correspondence mappings for a nondegenerate saddle satisfy the Pfaffian equations presented in Table 1. where $S_{0}$ denotes a nonresonant saddle and $S_{\mu}$ denotes a saddle of multiplicity $\mu$; see formula (2.2) for the definition of $P_{\mu}$.

TABle 1. Pfaffian equations for saddles

\begin{tabular}{|c|c|}
\hline Type & Pfaffian equations \\
\hline$S_{0}$ & $x d y-\lambda y d x=0$ \\
\hline$S_{\mu}$ & $P_{\mu}\left(x^{\mathrm{m}}, \lambda\right)\left[y P_{\mu}\left(y^{\mathrm{n}}, \lambda\right) d x-\left(\frac{\mathrm{n}}{\mathrm{m}}+P_{\mu}\left(y^{\mathrm{n}}, \lambda\right)\right) x P_{\mu}\left(x^{\mathrm{m}}, \lambda\right) d y\right]=0$ \\
\hline
\end{tabular}

There are two types of correspondence mappings for saddle-nodes: along the central manifold (see Figure 4) and from the hyperbolic manifold to the central one (or backwards). The correspondence mappings related to a saddle-node of multiplicity $\mu$ are denoted by $D_{\mu}^{c}$ and $D_{\mu}^{h}$.

Lemma 2.6 (Il'yashenko and Yakovenko [4). The correspondence mappings for the saddle-node satisfy the equations presented in Table 2] see formula (2.2) for the definition of $Q_{\mu}$.

TABLE 2. Pfaffian equations for saddle-nodes

\begin{tabular}{|c|c|}
\hline Type & Pfaffian equations \\
\hline$D_{\mu}^{c}$ & $x(x d y-y d x)=0$ \\
\hline$D_{\mu}^{h}$ & $Q_{\mu}(x, \lambda) d y-y d x=0$ \\
\hline
\end{tabular}

Remark 2.7. The factors $P_{\mu}\left(x^{\mathrm{m}}, \lambda\right)$ and $x$ in the Pfaffian equations for $S_{\mu}$ (see Table 1) and $D_{\mu}^{c}$ (see Table 2), respectively, are added in order to fulfill the topological requirements of Khovanskii's procedure (see 4, Lemma 3.1] for the details).

Remark 2.8. Obviously, the correspondence mappings for saddles of both types and saddle-nodes of type $D_{\mu}^{h}$ are defined for the unperturbed system and also for any sufficiently small perturbation of the system. This is not true for saddle-nodes of type $D_{\mu}^{c}$ : the correspondence mapping is defined only for certain perturbations of the initial system. (See Figure 4, the correspondence mapping is defined only for case $c$ ).)

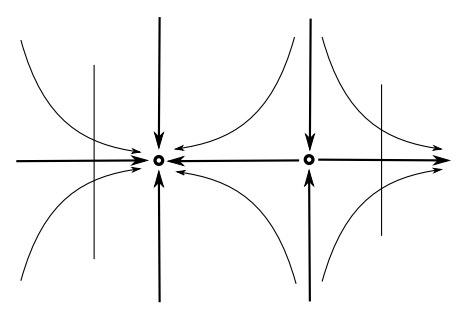

a)

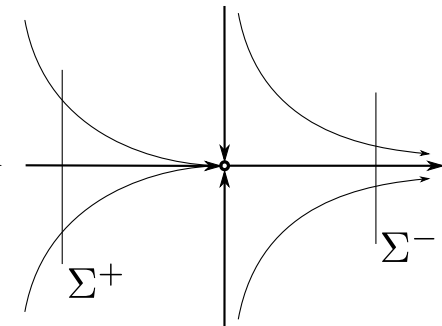

b)

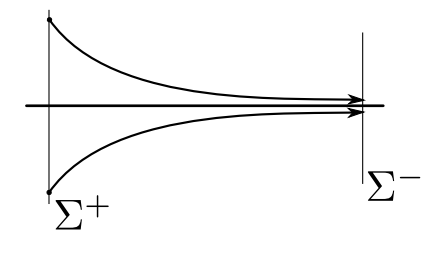

c)

FiguRE 4. The correspondence mapping for a saddle-node of type $D_{\mu}^{c}$ : a) for a precritical value of the parameter; $b$ ) for a critical value of the parameter; c) for a postcritical value of the parameter. 
Remark 2.9. The basic system for a monodromic polycycle makes sense for all values of the parameter for which all correspondence mappings are defined. However, the corresponding Pfaffian equations still make sense for any perturbations. Therefore, passage to the Pfaffian system can yield "redundant" solutions (not corresponding to limit cycles), but we do not lose the "useful" solutions (corresponding to limit cycles).

As has already been noted, we formally replace the singular equations in the basic system by the corresponding Pfaffian equations of the form

$$
A_{j} d x_{j}+B_{j} d y_{j}=0
$$

taken from Tables 1 and 2, obtaining the functional-Pfaffian system

$$
\begin{gathered}
\left\{\begin{array}{l}
\omega_{j}=0, \\
F_{j}(x, y, \varepsilon)=0,
\end{array} \quad j=1, \ldots, n,\right. \\
\omega_{j}:=A_{j} d x_{j}+B_{j} d y_{j}, \quad F_{j}(x, y, \varepsilon):=x_{j+1}-f_{j}\left(y_{j}, \varepsilon\right), \\
(x, y):=\left(x_{1}, y_{1}, \ldots, x_{n}, y_{n}\right) \in\left(\mathbb{R}^{2 n}, 0\right), \quad \varepsilon \in\left(\mathbb{R}^{k}, 0\right) .
\end{gathered}
$$

This system should be interpreted as follows: we take an arbitrary integral manifold $\Gamma$ of the system consisting of the Pfaffian equations in the system (2.5) and find the intersection of $\Gamma$ with the level set $F^{-1}(0)$, where

$$
F:\left(\mathbb{R}^{2 n}, 0\right) \rightarrow \mathbb{R}^{n}
$$

is the mapping with the coordinate functions $F_{j}$. The solutions of the basic system (2.1) yield a solution of the functional-Pfaffian system for a certain specific integral manifold $\Gamma$. Estimating the number of isolated intersection points for an arbitrary manifold $\Gamma$, we obtain an upper bound for the number of solutions of the basic system.

Limit cycles correspond to isolated fixed points of the Poincare map, so that we must estimate only the number of isolated solutions of (2.1). The latter number does not exceed the number of nondegenerate solutions of a similar system with a small right-hand side (see 4, Lemma 3.2]). In turn, these solutions correspond to transversal intersections of $\Gamma$ with a typical fiber $F^{-1}(a)$ for a small $a \in\left(\mathbb{R}^{n}, 0\right)$ (see [4, Lemma 3.3]).

Since the integral manifold and the level set have complementary dimensions, it follows that the transversal intersection always consists of isolated points, which are called regular solutions of system (2.5). We must estimate their number from above, and the estimate must be uniform with respect to all integral manifolds $\Gamma$ and all sufficiently small values of parameters.

In the further arguments, it is convenient to regard the parameters $\varepsilon$ and

$$
\lambda=\left(\lambda^{1}, \ldots, \lambda^{n}\right), \quad \lambda^{j} \in \mathbb{R}^{\mu_{j}+1},
$$

as additional independent variables. For this, we add to the system new equations of the form

$$
\varepsilon=\varepsilon^{*} \in \mathbb{R}^{k}, \quad \lambda=\lambda^{*} \in \mathbb{R}^{m},
$$

where $m:=n+\sum \mu_{j}$.

Furthermore, the degrees of the variables $x$ and $y$ in the Pfaffian equation for the resonant saddle $S_{\mu}$ (see Table 1) are unbounded because of the presence of the resonance $m: n$. The unbounded degrees are an obstruction to our research, since the degrees of the Pfaffian equations enter the final estimate. However, this is easy to correct by adding new variables,

$$
z=x^{\mathrm{m}} \quad \text { and } \quad w=y^{\mathrm{n}},
$$


and Pfaffian equations for $z$ and $w$. As a result, we obtain three Pfaffian equations for each resonant saddle:

$$
\left\{\begin{array}{l}
P_{\mu}(z, \lambda)\left[y P_{\mu}(w, \lambda) d x-\left(\frac{\mathrm{n}}{\mathrm{m}}+P_{\mu}(w, \lambda)\right) x P_{\mu}(z, \lambda) d y\right]=0, \\
x d z-\mathrm{m} z d x=0, \\
y d w-\mathrm{n} w d y=0 .
\end{array}\right.
$$

The first equation is said to be basic, and the other two are said to be additional. The equations in Table 2 are also said to be basic.

So, now we replace the functional-Pfaffian system (2.5) by a new system of the same type with the following distinctions:

(1) each Pfaffian equation for $S_{\mu}$ in Table 1 is replaced by the three equations (2.6);

(2) the parameters $\varepsilon$ and $\lambda$ in the expression (2.2) for $Q_{\mu}$ are included into the number of the variables, and the system contains equations for the parameters:

$$
\varepsilon-\varepsilon^{*}=b_{\varepsilon} \quad \text { and } \quad \lambda-\lambda^{*}=b_{\lambda},
$$

where the right-hand sides are small.

We look for an estimate not only for systems with zero right-hand side, but also for those with a small right-hand side. The estimate must be uniform with respect to the right-hand side.

The modified functional-Pfaffian system can be written as follows:

$$
\left\{\begin{array}{l}
\Omega=0 \backsim n+2 s, \\
F_{f}=a \backsim n, \\
F=b \backsim k+m .
\end{array}\right.
$$

Here, the line $\Omega=0$ denotes Pfaffian equations taken from Tables 1 and 2 , and from system (2.6) in the case of a resonant saddle. The line $F_{f}=a$ denotes functional equations of the form

$$
x_{j+1}-f_{j}\left(y_{j}, \varepsilon\right)=a_{j},
$$

while the line $F=b$ denotes equations for the parameters $\varepsilon$ and the additional parameters $\lambda$. The numbers to the right of the sign " $\backsim$ " mean a bound for the number of the equations of the type indicated. The phase space of the system has dimension

$$
\mathbf{m}:=2 n+2 s+k+m .
$$

2.5. Khovanskiř's procedure. A detailed description of Khovanskiı̌'s procedure [6] is beyond the scope of the present paper. In this section, we mention only the most necessary facts (see [5, §3.3]).

Khovanski1's procedure consists in sequential replacement of the Pfaffian equations (one at each step) by functional equations in accordance with a certain algorithm. Each step of the procedure consists in passing from a system of the form (2.7) to one or two similar systems with a smaller number of Pfaffian equations. Furthermore, the number of solutions of the initial system does not exceed the sum of the numbers of solutions of the new systems.

As a result of the procedure, we pass from a single functional-Pfaffian system to a collection of purely functional systems of a special form, for which the number of solutions is estimated with the help of an analog of Bezout's theorem. Furthermore, the sum of the bounds for the numbers of solutions of the systems estimates the number of solutions of the initial functional-Pfaffian system.

In more detail, the algorithm is as follows. At each step of the procedure, one of the Pfaffian equations in the initial system is replaced either by a functional contact equation or by an equation with a covering function. 
The contact equation has the form

$$
\mathcal{F}:=* \omega_{1} \wedge \cdots \wedge \omega_{n+2 s} \wedge d F_{f_{1}} \wedge \cdots \wedge d F_{f_{l}} \wedge d F_{1} \wedge \cdots \wedge d F_{k}=\epsilon
$$

(see [5, $\S 3.3$ and formula (47)]), where the $\omega_{j}$ are the left-hand sides of the Pfaffian equations,

$$
F_{f_{j}}:=y_{j}-f_{j}\left(x_{j}, \varepsilon\right), \quad F_{j}:=\varepsilon_{j}-b_{j},
$$

and the number $\epsilon$ is small.

The equation with a covering function has the form

$$
\rho:=\rho_{1} \cdots \rho_{n} \rho_{\varepsilon}=\epsilon
$$

(see [5, formula (64)]), where

$$
\rho_{\varepsilon}=\sum\left(r^{2}-\varepsilon_{i}^{2}\right)
$$

for a certain small $r$, the functions $\rho_{j}$ are taken from Table 3 in accordance with the type of the $j$ th singular point, and $\epsilon$ is a small number.

TABLE 3. Covering functions

\begin{tabular}{|c|c|}
\hline Type of the singular point & Covering function \\
\hline$S_{0}$ & $x y(r-x)(r-y) \widetilde{\rho}$ \\
\hline$S_{\mu}$ & $x y z w(r-x)(r-y)(r-z)(r-w) P_{\mu}^{2}(z, \lambda) \widetilde{\rho}$ \\
\hline$D_{\mu}^{c}$ & $x^{2}\left(r^{2}-x^{2}\right)\left(r^{2}-y^{2}\right) \widetilde{\rho}$ \\
\hline$D_{\mu}^{h}$ & $y(r-y)\left(r^{2}-x^{2}\right) Q_{\mu}(x, \lambda) \widetilde{\rho}$ \\
\hline
\end{tabular}

Explanations to Table 3. The table presents covering functions for various types of singular points. Here, $r$ is a small parameter, $z:=x^{\mathrm{m}}$, and $w:=y^{\mathrm{n}}$. Finally, $\widetilde{\rho}$ is a polynomial of the form

$$
\widetilde{\rho}(\lambda):=\left(r^{2}-\lambda_{1}^{2}\right) \cdots\left(r^{2}-\lambda_{\mu-1}^{2}\right)\left(r^{2}-\left(c-\lambda_{\mu}\right)^{2}\right),
$$

where $c$ is a real number called the localization parameter of the family. (See [3] for more details.) The rest of the notation was introduced before (see Subsection 2.3).

Thus, two new systems arise, each containing one differential equation less than the original system. After the replacement of one equation, the next equation is replaced in a similar way. However, if at a certain step the system already contains an equation with a covering function, then afterwards the Pfaffian systems are replaced by contact equations only (see the plan).
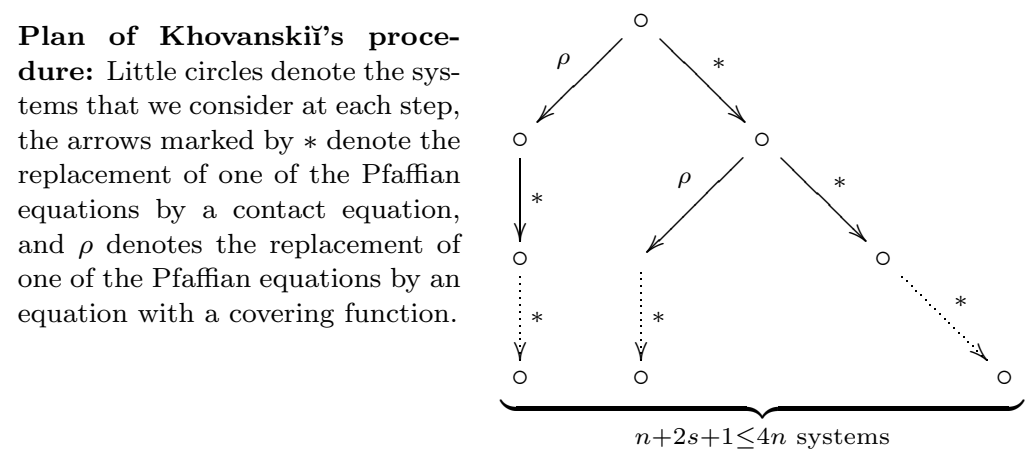

Khovanskil's procedure stops when all Pfaffian equations in the system are replaced. Since the number of Pfaffian equations is equal to $n+2 s$, we obtain $n+2 s+1 \leq 4 n$ systems as a result. 
Assertion 2.10 (See Theorem 7 and Corollary 4 in [5). After the passage from a single system to one or two new systems (as indicated above), the total number of solutions of all resulting systems majorizes the number of solutions of the original system.

Remark 2.11. Let $\mathcal{G}_{\mathbf{f}}=a$ be a system obtained at a certain step of Khovanskií's procedure. Taking (2.8) and (2.9) into account, we easily show by induction that each component $\mathcal{G}_{i}$ is either a polynomial in the variables, the parameters, the functions $f_{j}$, and their derivatives of various orders, or a differential form with polynomial coefficients.

Remark 2.11 motivates the following definition.

Definition 2.12. Consider an equation in the system involved in Khovanskiı's procedure. If the equation is Pfaffian, it has the form $\omega=0$. The $K$-degree of the equation is the maximum degree of the coefficients of the form $\omega$. If the equation is functional, then its $K$-degree is the degree of the equation as of a polynomial in the variables, the functions $f_{j}$, and their derivatives.

Remark 2.13. The K-degree of any Pfaffian equation is equal to the usual degree of the equation; the same is also true for any covering function. The K-degree of each component of the functional equation $F_{f}=a$, as well as the degree of the differential of the component, is equal to unity. The K-degree of each component of the equation for the parameters is also equal to unity, while the K-degree of the differential of the component is zero.

Applying Khovanski1's procedure to system (2.7), we obtain at most $4 n$ functional systems, and we can estimate the K-degree of each system (see 3 ). The number of solutions of the initial system does not exceed the sum of the numbers of solutions of the final systems. Should the latter systems be only polynomial (with respect to the variables), to estimate the number of solutions it would have sufficed to apply the classical Bezout theorem.

It turns out that, under certain conditions on the right-hand side of the functional system, an analog of Bezout's theorem holds (see Theorem 2.15). This analog yields an estimate of the number of solutions for typical Cartesian mappings by the product of the $\mathrm{K}$-degrees of all equations in the system.

Using Theorem 2.15, we estimate the number of solutions of the systems obtained by applying Khovanski1's procedure. After that, we multiply the result by the number of the systems, thus estimating the number of solutions of the initial system.

2.6. Bezout-Kaloshin theorem on the estimate for the number of solutions and its application. We formulate the Bezout-Kaloshin theorem not in full generality, but in the form in which we shall use it. The complete formulation can be found in 5 , Theorem 12].

Let

$$
\mathcal{G}_{\mathbf{f}}=a
$$

be a certain system obtained by Khovanskiı̌'s procedure. Here, we have

$$
\mathcal{G}_{\mathbf{f}}:=\left(P^{1}, \ldots, P^{\mathbf{m}}\right),
$$

where the $P^{i}$ are polynomials in the variables, the functions $f_{j}$, and their derivatives. We observe that if $i \neq j$, then the functions $f_{i}$ and $f_{j}$ depend on distinct collections of variables.

Let $f_{i} \in C_{\mathcal{I}_{i}}^{p}$, where $p>\mathbf{m}+1$ and $\mathcal{I}_{i}$ denotes the collection of the variables corresponding to the function $f_{i}$. 
Definition 2.14. For any positive integer $d$ and small positive $\delta$, the set

$$
K^{d, \delta}:=\left\{\left(a_{1}, \ldots, a_{\mathbf{m}}\right): 0<\left|a_{1}\right|<\delta, 0<\left|a_{j+1}\right|<\left|a_{1} \cdots a_{j}\right|^{d}, j=1, \ldots, \mathbf{m}-1\right\}
$$

is called the Kaloshin cone.

Theorem 2.15 (Bezout-Kaloshin; see [5]). There exist an open, everywhere dense set $F_{P}$ in the function space

$$
C_{\mathcal{I}_{1}}^{p} \times \cdots \times C_{\mathcal{I}_{n}}^{p}
$$

and an integer $d$ such that for each $\mathbf{f} \in F_{P}$ there are a characteristic size $r_{0}>0$ and a number $\delta>0$ for which the number of regular preimages of an arbitrary point $a=$ $\left(a_{1}, \ldots, a_{\mathbf{m}}\right)$ in the Kaloshin cone $K^{d, \delta}$ admits the estimate

$$
\#\left\{x \in U_{r}: \mathcal{G}_{\mathrm{f}}=a\right\} \leq \prod_{1}^{\mathbf{m}} \operatorname{deg} P^{i},
$$

where $0<r \leq r_{0}$, and $U_{r} \subset \mathbb{R}^{\mathbf{m}}$ is the $r$-cube centered at the origin.

The following lemma, the proof of which is contained in $\S 4$ of the paper [5], allows us to apply the Bezout-Kaloshin theorem.

Lemma 2.16. We may assume that the right-hand sides $a:=\left(a_{1}, \ldots, a_{\mathbf{m}}\right)$ of the functional systems obtained by Khovanskiu's procedure belong to the Kaloshin cone in the statement of Theorem 2.15 .

As was already noted at the end of Subsection 2.5, after applying the Bezout-Kaloshin theorem it only remains to estimate the degrees of the systems obtained by Khovanski1̌'s procedure.

Now we can rigorously formulate what the term genericity means.

We order the systems obtained by Khovanski1̌'s procedure in an arbitrary way and denote by $F_{P_{j}}$ the open, everywhere dense set in Theorem 2.15 for the $j$ th system.

Definition 2.17. A collection $\mathbf{f}=\left(f_{1}, \ldots, f_{n}\right)$ consisting of $C^{p}$-smooth functions, $p>$ $\mathbf{m}+1$, is said to be generic if $\mathbf{f} \in F_{P_{j}}$ for all $j=1, \ldots, \mathbf{m}$.

2.7. Differences in approaches. $\mathrm{Il}^{\prime}$ yashenko and Yakovenko 4 investigated the number of the regular preimages of points in the image (see system (2.5) and its description on p. 564) without any restrictions on the points. Using Khovanskiı's method and Gabrielov's theorem, they proved that $E(k)$ is finite for any positive integer $k$.

In [5, Kaloshin considered only preimages of special points of the image (see the condition on $a_{i}$ in the statement of Theorem 2.15). This allowed him to prove an analog of Bezout's theorem (see Theorem 2.15), which simplified the analysis the result of which was finding an explicit estimate for the number $E(k)$.

In the present paper, we use the same method. However, a more accurate combinatorial analysis yields an explicit estimate for $E(n, k)$. The main idea of the calculation is to control the growth of the degrees at each step of Khovanski1's procedure and to give a common estimate for the degrees of the equations in the systems at each step.

The difference between our paper and Kaloshin's paper [5] consists in the method of calculating the degrees at the penultimate step in the plan of the proof (the dotted arrow in Figure 2). 


\section{§3. UPPER ESTimate FOR THE NUMBeR $E(n, k)$}

In this section, we perform the computations needed for the proof of Theorem 1.8

We recall our notation:

$k$ is the number of parameters of the family (the codimension);

$n$ is the number (with repetitions) of vertices in the polycycle;

$s$ is the number of resonant saddles;

$\mu_{j}$ is the multiplicity of the $j$ th singular point; and

$m:=n+\sum \mu_{j}$ is the dimension of the space of parameters $\lambda$.

We consider the functional-Pfaffian system

$$
\begin{cases}\Omega=0 & \sim n+2 s \leq 3 n, \\ F_{f}=a & \sim n, \\ F=b & \sim k+m .\end{cases}
$$

Applying Khovanski1's procedure to this system, we obtain $4 n$ functional systems as a result. By the Bezout-Kaloshin theorem, in order to estimate the number of solutions to such a system, it suffices to estimate the product of the K-degrees of the equations in the system.

For this, we first estimate the K-degrees of system (3.1), of the contact function for this system, and of the covering function. After that, using the results obtained, we estimate the growth of the K-degrees in Khovanski1's procedure (see Lemma 3.4). Finally, we use the latter estimate to obtain the required result.

Remark 3.1. The degree of the polynomial coefficients of the equations in the group $\Omega=0$ of the system (2.7) (also see (3.1)) does not exceed $6 \mu+4$.

Proof. For instance, we consider the Pfaffian equation for the resonant saddle $S_{\mu}$ (see Table 1). Since $\operatorname{deg} P_{\mu}=2 \mu+1$ (see (2.2) ), the degree of the Pfaffian equation in the variables $x, y, z=x^{\mathrm{m}}, w=y^{\mathrm{n}}$, and $\lambda$ is equal to $6 \mu+4$. The remaining equations are treated in a similar way.

Lemma 3.2. The following assertions hold true for the functional-Pfaffian system (3.1):

1) the $K$-degree of the contact function $\mathcal{F}$ does not exceed $6 k+7 n$, and

$2)$ the $K$-degree of the differential $d \mathcal{F}$ does not exceed that of $\mathcal{F}$.

Proof. 1) We recall the expression for the contact function (see Subsection 2.5):

$$
\mathcal{F}=* \omega_{1} \wedge \cdots \wedge \omega_{n+2 s} \wedge d F_{f_{1}} \wedge \cdots \wedge d F_{f_{l}} \wedge d F_{1} \wedge \cdots \wedge d F_{k} .
$$

Here, the $\omega_{j}$ are the left-hand sides of the Pfaffian equations,

$$
F_{f_{j}} \equiv y_{j}-f_{j}\left(x_{j}, \varepsilon\right) \quad \text { and } \quad F_{j}=\varepsilon_{j}-b_{j} .
$$

The forms $\omega_{j}$ corresponding to the additional equations of resonant saddles have unit $\mathrm{K}$-degree, and their number is $2 s \leq 2 n$. In accordance with Remark 3.1, the K-degree of the remaining forms $\omega_{j}$ is estimated by $6 \mu_{j}+4$, and their number is $n$. Observe that

$$
\sum \mu_{j} \leq k
$$

The forms $d F_{f_{j}}$ have K-degree 1 , while the forms $d F_{j}$ have K-degree equal to zero. Combining all estimates, we obtain the first assertion of the lemma.

2) The assertion concerning the K-degrees of the differential holds true not only for the contact function but also for any polynomial in the variables, the functions $f_{j}$, and their derivatives. 
Indeed, differentiation of a polynomial with respect to any variable reduces the degree of the polynomial by one. Differentiation with respect to the variables $x_{j}, \varepsilon$, and $\lambda$ can also yield a factor of the form $\partial f_{j} / \partial x_{j}$, for example:

$$
F=f_{1}\left(x_{1}, y_{1}, \varepsilon\right), \quad \frac{\partial F}{\partial x_{1}}=\frac{\partial f_{1}\left(x_{1}, y_{1}, \varepsilon\right)}{\partial x_{1}} .
$$

As a result, the K-degree of the polynomial is preserved. This completes the proof of the lemma.

Remark 3.3. We easily see that:

(1) the degree of any covering function in Table 3 is estimated by the expression

$$
\operatorname{deg} \rho_{j} \leq 6 \mu+10
$$

(2) the degree of the covering function in Khovanskiu's procedure (see equation (2.9)) is estimated by the expression

$$
\operatorname{deg} \rho \leq 8 k+10 n .
$$

Proof. Inequality (3.2) follows from the relations

$$
\operatorname{deg} P_{\mu}=2 \mu+1, \quad \operatorname{deg} Q_{\mu}=2 \mu+2, \quad \text { and } \quad \operatorname{deg} \widetilde{\rho}_{\mu}=2 \mu
$$

(see (2.2) and (2.10) $)$. Inequality (3.3) follows from (3.2), (2.9), and $\sum \mu_{j} \leq k$.

Lemma 3.4 (On the exponential growth of degrees). The K-degree of the contact equation and the covering function (if the latter occurs) at the jth step of Khovanskiu's procedure does not exceed $2^{j} D$, where $D:=18 k$.

Proof. We observe that, by the inequality $n \leq k$, for any positive integer $k$ we have the inequality

$$
D \geq \max (6 k+7 n, 8 k+10 n) .
$$

For $j=1$, the lemma follows from inequality (3.4), Lemma 3.2, and Remark 3.1.

Now, assuming that the claim holds true for all $j<j_{0}$, we prove it for $j_{0}$. Observe that the sum of the K-degrees of the equations in the initial system does not exceed $D$. At the $j_{0}$ th step, we have two possibilities: the next form is replaced by either the covering function or the contact equation.

In the first case, once again, the claim follows from Remark 3.1. We consider the contact equation at the $j_{0}$ th step of Khovanskiı's procedure. By construction, the K-degree of the equation does not exceed the sum of the K-degrees of the equations in the system at the preceding step, for which the inductive hypothesis holds true. This sum has the form

$$
\sum_{1}^{j_{0}-1} 2^{j} D+D=2^{j_{0}} D
$$

Proof of Theorem 1.8. Let $\Pi$ be the product of the K-degrees of the equations in each system obtained as a result of Khovanski1's procedure. Using the lemma on the exponential growth of degrees, we write the following chain of inequalities:

$$
\Pi \leq \prod_{j=1}^{n+2 s} 2^{j} D \leq \prod_{j=1}^{3 n} 2^{j} D \leq 2^{5 n^{2}+2 n} D^{3 n} \leq 2^{5 n^{2}+17 n} \cdot k^{3 n} .
$$

\footnotetext{
${ }^{3}$ Indeed, a saddle-node is a degeneration of codimension one, while coincidence of the separatrices of two saddles is a degeneration of the same codimension. Therefore, a polycycle with $n$ vertices is a degeneration of codimension at least $n$.
} 
In total, Khovanski1's procedure yields at most $4 n$ systems. Applying the BezoutKaloshin theorem to them, we obtain the announced result: we have

$$
E(n, k) \leq 2^{5 n^{2}+20 n} k^{3 n} \text {. }
$$

Theorem 1.8 is proved.

\section{REFERENCES}

[1] V. Arnol'd, V. Afraǐmovich, Yu. Ilyashenko, and L. Shil'nikov, Bifurcation theory, Itogi Nauki i Tekhniki Sovrem. Probl. Mat. Fundam. Naprav., vol. 5, Dynamical Systems-5, VINITI, Moscow, 1986, pp. 5-218; English transl., Encyclopaedia Math. Sci., vol. 5, Springer-Verlag, Berlin, 1994, pp. 1-205. MR0895653

[2] Yu. Ilyashenko, Normal forms for local families and nonlocal bifurcations, Astérisque No. 222 (1994), 233-258. MR 1285390 (95g:58180)

[3] Yu. Ilyashenko and S. Yakovenko, Finitely smooth normal forms of local families of diffeomorphisms and vector fields, Uspekhi Mat. Nauk 46 (1991), no. 1, 3-39; English transl., Russian Math. Surveys 46 (1991), no. 1, 1-43. MR.1109035 (92i:58165)

[4] _ Finite cyclicity of elementary polycycles in generic families, Concerning the Hilbert 16th Problem, Amer. Math. Soc. Transl. Ser. 2, vol. 165, Amer. Math. Soc., Providence, RI, 1995, pp. 2195. MR:1334340 (96f:34042)

[5] V. Kaloshin, The existential Hilbert 16th problem and an estimate for cyclicity of elementary polycycles, Invent. Math. 151 (2003), no. 3, 451-512. MR.1961336(2004e:34054)

[6] A. G. Khovanskiǔ, Fewnomials, FAZIS, Moscow, 1997; English transl., Transl. Math. Monogr., vol. 88, Amer. Math.Soc., Providence, RI, 1991. MR1619432 (99f:14070) MR1108621 (92h:14039)

[7] R. Roussarie, A note on finite cyclicity property and Hilbert's 16th problem, Dynamical Systems (Valparaiso, 1986) (R. Bamon, R. Lavarca, J. Palis, eds.), Lecture Notes in Math., vol. 1331, SpringerVerlag, Berlin, 1988, pp. 161-168. MR0961099 (90b:58227)

OJSC "N. A. Dollezhal Research and Development Insitute of Power Engineering", M. Krasnoselskaya 2/8, Moscow 107140, Russia

E-mail address: pkaleda@yandex.ru

National Research University Higher School of Economics, Kochnovsky 3, Moscow, Russia E-mail address: ilya.schurov@noo.ru

Received 5/JUL/2009

Translated by N. YU. NETSVETAEV 